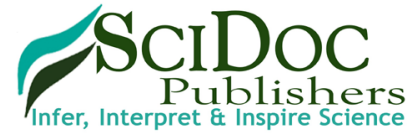

\section{International Journal of Ophthalmology \& Eye Science (IJOES) \\ ISSN 2332-290X}

\title{
Posterior Uveal Melanoma
}

Cham $\mathrm{KM}^{1^{*}}$, Tram L ${ }^{2}$

${ }^{1}$ Department of Optometry and Vision Sciences, The University of Melbourne, Victoria, Australia.

${ }^{2}$ OPSM Smithfield, Cairns QLD, Australia.

\section{Abstract}

Uveal melanoma is a deadly ocular malignancy that involves predominantly the choroid. Once diagnosed, patients usually require long-term follow up to detect any local recurrence and metastatic disease to the liver, lung and skin. One must be competent at diagnosing choroidal melanoma in order to enable prompt intervention, which is critical to patient survival. When faced with the challenge of distinguishing it from an initial choroidal nevus or a nevus potentially transforming into a melanoma, especially small-sized, indicators for malignancy include presence of orange lipofuscin pigment, associated sub-retinal fluid, tumour proximity within $3 \mathrm{~mm}$ of the optic disc, absence of halo or drusen, and symptoms of flashes, floaters or blurred vision.

A comprehensive dilated fundus examination supplemented with fundus imaging such as optical coherence tomography and fundus auto-fluorescence is warranted. Ancillary testing using fluorescein and indocyanine green angiography, and ultrasonography demonstrating tumour hollowness in malignancy may also prove beneficial.

Keywords: Choroidal; Uvea; Tumour; Melanoma; Cancer.

\section{Introduction}

Uveal melanoma is a fatal intra-ocular malignant tumour with risk for metastasis that affects men in $51 \%$, and mainly Caucasians (98\%). The mean age at patient presentation is 60 years and the melanoma involve primarily the choroid (90\%) [1]. The tumour may appear as a deep retinal mass without feeder vessels, together with retinal detachment or vitreous haemorrhages [2]. We present a patient with a choroidal melanoma treated with brachytherapy.

\section{Case Report}

A 76-year-old asymptomatic male attended the clinic in 2014 requesting a routine review of a left choroidal melanoma that was detected post-cataract operation in 2010. Subsequently he was treated with brachytherapy delivered with a ruthenium-106 plaque. Since then, there has been no evidence of metastatic disease and the tumour remains inactive.
Upon examination, his best corrected visual acuities were $6 / 7.5$ in the right eye and $6 / 12+$ in the left eye with no improvement in pinhole testing. Anterior segment biomicroscopy revealed clear and centred acrylic intraocular lens and normal intraocular pressure. Dilated fundus examination revealed a pigmented choroidal melanoma in the supero-temporal peripheral retina with intraocular haemorrhages and sub-retinal fluid track (Figure 1). Concerned about possible signs of tumour regression and radiation retinopathy, the patient was referred back to his previous ophthalmologist who had treated his melanoma for a second opinion. The Ophthalmologist later confirmed the melanoma to be dry and inert with no overlying fresh orange lipofuscin pigment that would otherwise indicate active tumour activity. The sub-retinal fluid and intraocular haemorrhages were not deemed to be clinically significant and he was placed under observation. Optos Fundus auto-fluorescence (AF) Opto-map image demonstrates the typical clinical presentation of a previously treated choroidal melanoma (Figure 2).

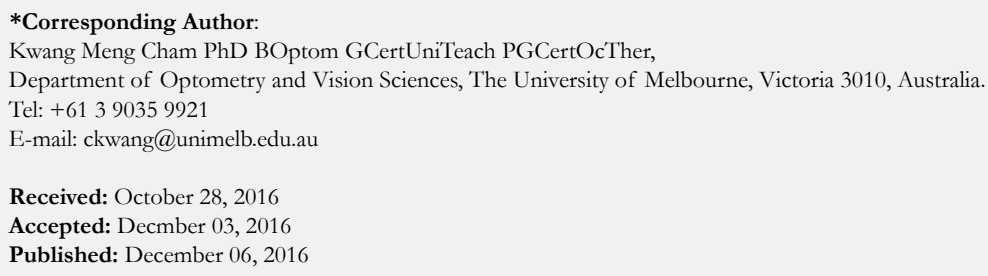

Copyright: Cham $\mathbf{K M}^{\circ}$ 2016. This is an open-access article distributed under the terms of the Creative Commons Attribution License, which permits unrestricted use, distribution and reproduction in any medium, provided the original author and source are credited. 
Figure 1. Optos Opto-Map Image Of the Pigmented Choroidal Melanoma Four Years After Radiotherapy. Small Intraocular haemorrhages Were Observed at Time of Presentation.

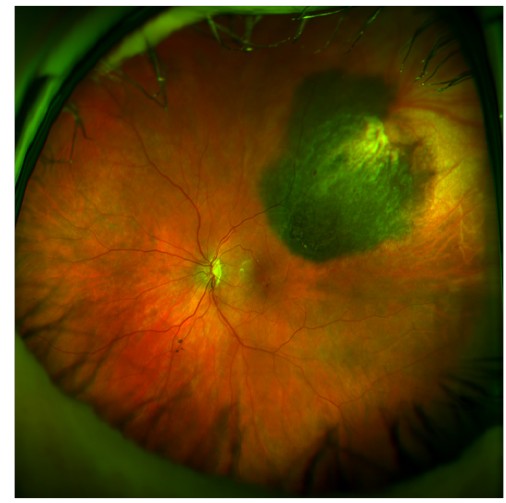

Figure 2. Optos AF Opto-mapimage of the Treated Choroidal Melanoma. On AF, the tumour displays marked mottled hypo-AF interspersed mild hyper-AF from increased retinal pigment epithelium pigment (RPE) and fibrous metaplasia over time [3]. An old track of sub-retinal fluid (hyper-AF) can be seen inferior to the tumour.

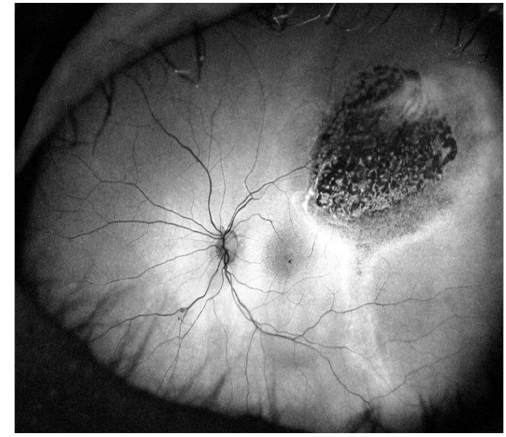

\section{Discussion}

The clinical features of choroidal melanoma varies in pigmentation, and factors such as increasing age, diffuse configuration, large tumour size involving the ciliary body, presence of sub-retinal fluid, intraocular haemorrhage, and extra-ocular extension can result in poor prognosis [4]. We could not classify the choroidal melanoma for prognostication based on the Tumour, Node, and Metastasis staging system of the American Joint Committee on Cancer due to lack of tumour dimensions (basal diameter and thickness, size and height) [5, 6]. After considering patient's age, systemic and ocular status such as tumour size and location, treatment options may include transpupillary thermotherapy, charged particle irradiation, local resection, orbital extenteration, enucleation and focal radiotherapy (plaque or proton beam), with the latter two being the most common therapeutic options [2].

Differentials of choroidal melanoma include peripheral exudative haemorrhagic chorioretinopathy $(8 \%)$, congenital hypertrophy of the RPE $(6 \%)$, idiopathic haemorrhagic detachment of the retina or RPE (5\%), circumscribed choroidal hemangioma (5\%), age-related macular degeneration (4\%), and choroidal nevus (49\%), which is most commonly misdiagnosed when differentiating from small melanomas, and, yet plays an important role in uveal melanoma development [2].

One must be familiar with the characteristics of a choroidal melanoma in order to enable accurate diagnosis and early therapeutic intervention, which is imperative to patient survival. When faced with the challenge of distinguishing it from an initial choroidal nevus or a nevus potentially transforming into a melanoma, especially small-sized, indicators for malignancy include presence of orange lipofuscin pigment, associated sub-retinal fluid, tumour proximity within $3 \mathrm{~mm}$ of the optic disc, absence of halo or drusen, and symptoms of flashes, floaters or blurred vision [7]. Therefore a comprehensive dilated fundus examination supplemented with fundus imaging such as optical coherence tomography and fundus auto-fluorescence is warranted. Ancillary testing using fluorescein and indocyanine green angiography, and ultrasonography demonstrating tumour hollowness in malignancy may also prove beneficial.

\section{Conclusion}

The prognosis for this patient is favourable currently since the melanoma has remained stable, but he will need long-term follow up to detect any local recurrence and metastatic disease to the liver, lung and skin. The tumour needs to be monitored by an ocular oncologist two to three times yearly. Systemic evaluation every six months consisting of liver function and imaging procedures, and chest radiograph, should be recommended for patients with choroidal melanoma.

\section{References}

[1]. Shields CL, Kaliki S, Furuta M, Mashayekhi A, Shields JA (2012) Clinical spectrum and prognosis of uveal melanoma based on age at presentation in 8033 cases. Retina. 32(7): 1363-1372. DOI: 10.1097/ IAE.0b013e31824d09a8.

[2]. Shields CL, Manalac J, Das C, Ferguson K, Shields JA (2014) Choroidal melanoma: clinical features, classification, and top 10 pseu- 
domelanomas. Curr Opin Ophthalmol 25(3): 177-185.DOI:10.1097/ ICU.0000000000000041.

[3]. Lavinsky D, Belfort RN, Navajas E, Torres V, Martins MC, et al., (2007) Fundus autofluorescence of choroidal nevus and melanoma. Br J Ophthalmol. 91(10): 1299-1302. DOI:10.1136/bjo.2007.116665

[4]. Shields CL, Furuta M, Thangappan A, Nagori S, Mashayekhi A, et al., (2009) Metastasis of uveal melanoma millimetre-by-millimetre in 8033 consecutive eyes. Arch Ophthalmol. 127(8): 989-998.DOI:10.1001/archophthalmol.2009.208.

[5]. Finger PT (2009) The $7^{\text {th }}$ edition AJCC staging system for eye cancer: an international language for ophthalmic oncology. Arch Pathol Lab Med.
133(8): 1197-1198. DOI: 10.1043/1543-2165-133.8.1197.

[6]. Shields CL, Kaliki S, Furuta M, Fulco E, Alarcon C, et al., (2013) American Joint Committee on Cancer classification of posterior uveal melanoma (tumor size category) predicts prognosis in 7731 patients. Ophthalmol. 120(10): 2066-2071.DOI: 10.1016/j.ophtha.2013.03.012.

[7]. Shields CL, Furuta M, Berman EL, Zahler JD, Hoberman DM, et al. (2009) Choroidal nevus transformation into melanoma: analysis of 2514 consecutive cases. Arch Ophthalmol. 127(8): 981-997. DOI:10.1001/archophthalmol.2009.151. 